\title{
Developing CAR T cell therapy
}

A new study reports the development of prostate cancer immunotherapy on the basis of chimeric antigen receptor (CAR) T cells. Injection of patient-derived $\mathrm{CD} 8^{+} \mathrm{T}$ cells that had been engineered to be specific for prostate-specific membrane antigen (PSMA) and insensitive to transforming growth factor- $ß$ (TGFß) into PSMA-expressing PC3 tumours in mice resulted in suppressed growth, as well as widespread apoptosis and T cell infiltration of tumours.

Current treatments for prostate cancer intended to elicit antitumour immune responses have limited efficacy. Now, researchers have created prostatetumour-specific CAR T cells by infecting $\mathrm{CD} 8^{+} \mathrm{T}$ cells from patients with metastatic disease with a retroviral construct. The construct carried an anti-PSMA chimeric immunoglobulin T cell receptor(ל) gene, conferring $T$ cell specificity, and a dominant negative TGFß type II receptor gene, which provides resistance to TGFß-mediated suppression of cytotoxic T cell function. The cells also expressed HSV1 thymidine kinase, enabling cell killing through ganciclovir treatment as a safety mechanism.

The CAR T cells could be expanded 23.4-fold in 21 days, which was not suppressed by TGFß, but ganciclovir decreased survival to $1.3 \%$ in 5 days. In a mouse xenograft model, two injections of the CAR T cells reduced the total burden of PSMA-expressing PC 3 tumours by $>82 \%$, but not that of normal PC 3 tumours. PC3-PSMA tumours showed evidence of nuclear fusion, fragmentation, necrosis, and apoptosis, as well as $\mathrm{CD} 8^{+} \mathrm{T}$ cell infiltration into the tumour parenchyma. In addition, mice that had received the PSMA-specific, TGFß-insensitive CAR T cells had increased serum IFN $\gamma$ and IL-2 levels in compared with animals that received naive $C D 8^{+} T$ cells, suggesting activation of local and systemic antitumour immunity.

This proof of principle study indicates this treatment as a potential new approach for men with metastatic prostate cancer.

Clemens Thoma

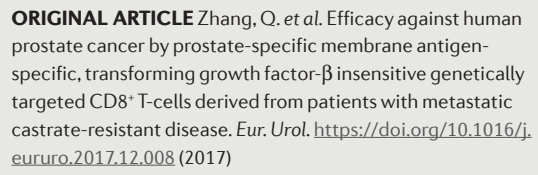

